\title{
Secondhand tobacco smoke exposure is associated with prolactin but not thyroid stimulating hormone among nonsmoking women seeking in vitro fertilization
}

\section{Citation}

Benedict, Merle D., Stacey A. Missmer, Kelly K. Ferguson, Allison F. Vitonis, Daniel W. Cramer, and John D. Meeker. 2012. "Secondhand Tobacco Smoke Exposure Is Associated with Prolactin but Not Thyroid Stimulating Hormone Among Nonsmoking Women Seeking in Vitro Fertilization." Environmental Toxicology and Pharmacology 34 (3) (November): 761-767. doi:10.1016/j.etap.2012.09.010.

\section{Published Version}

doi:10.1016/j.etap.2012.09.010

\section{Permanent link}

http://nrs.harvard.edu/urn-3:HUL.InstRepos:27336523

\section{Terms of Use}

This article was downloaded from Harvard University's DASH repository, and is made available under the terms and conditions applicable to Other Posted Material, as set forth at http:// nrs.harvard.edu/urn-3:HUL.InstRepos:dash.current.terms-of-use\#LAA

\section{Share Your Story}

The Harvard community has made this article openly available.

Please share how this access benefits you. Submit a story.

\section{Accessibility}




\title{
Secondhand tobacco smoke exposure is associated with prolactin but not thyroid stimulating hormone among nonsmoking women seeking in vitro fertilization
}

\author{
Merle D. Benedict $^{a}$, Stacey A. Missmer ${ }^{b, c, d}$, Kelly K. Ferguson $^{e}$, Allison F. Vitonis ${ }^{d}$, Daniel \\ W. Cramere, and John D. Meeker ${ }^{\mathrm{e}}$ \\ aDepartment of Safety, Health and Industrial Hygiene, Montana Tech of the University of \\ Montana, Butte, MT, USA \\ bDepartment of Epidemiology, Harvard School of Public Health, Boston, MA, USA \\ 'Department of Obstetrics, Gynecology and Reproductive Biology, Brigham and Women's \\ Hospital and Harvard Medical School, Boston, MA, USA \\ 'Channing Laboratory, Department of Medicine, Brigham and Women's Hospital and Harvard \\ Medical School, Boston, MA, USA \\ eDepartment of Environmental Health Sciences, University of Michigan, Ann Arbor, MI, USA
}

\section{Introduction}

Prolactin (PRL) and thyroid stimulating hormone (TSH; also known as thyrotropin) are important reproductive hormones. PRL is secreted by the anterior pituitary and was originally identified by its ability to stimulate mammary gland development and lactation. We now know that it is involved in over 300 separate actions in various vertebrates, including effects on reproduction, growth and development, metabolism, water and electrolyte balance, brain and behavior, and immunoregulation (Bole-Feysot et al., 1998). The largest group of actions for PRL pertains to reproductive processes.

TSH is also secreted by the anterior pituitary, and it stimulates the thyroid gland to produce and secrete thyroid hormones. TSH is regulated via negative feedback from thyroid hormones. Normal thyroid function is an important component of reproductive health. In females, thyroid dysfunction has been linked to menstrual disturbances, reduced fertility, spontaneous abortion and various late-pregnancy outcomes, including preterm birth and low birth weight (Krassas et al., 2010). Proper thyroid function is important to many other processes, as well, including energy balance, metabolism, and other functions in the nervous, cardiovascular, and pulmonary systems.

\footnotetext{
(C) 2012 Elsevier B.V. All rights reserved.

Address correspondence to: John Meeker, ScD, Department of Environmental Health Sciences, University of Michigan School of Public Health, 6635 SPH Tower, 109 S. Observatory St.. Ann Arbor, MI 48109, Phone: 1-734-764-7184, Fax: 1-734-763-8095, meekerj@umich.edu.

Publisher's Disclaimer: This is a PDF file of an unedited manuscript that has been accepted for publication. As a service to our customers we are providing this early version of the manuscript. The manuscript will undergo copyediting, typesetting, and review of the resulting proof before it is published in its final citable form. Please note that during the production process errors may be discovered which could affect the content, and all legal disclaimers that apply to the journal pertain.

Conflict of interests

The authors declare no competing interests.
} 
Studies have examined the effects of active smoking on TSH and thyroid function. McDonald et al. (2008) found that women who smoke during pregnancy had significantly lower TSH levels than nonsmokers. Triiodothyronine (T3) was not measured in that study, but free thyroxine (T4) concentrations did not differ between exposure groups, neither did cord blood TSH concentration from infants born of smokers compared to infants of nonsmokers (McDonald et al., 2008).

Shields et al. (2009) later confirmed some of these findings. For example, they also observed lower TSH concentrations in serum among pregnant smokers compared to nonsmokers and no significant difference in free $\mathrm{T} 4$ concentrations between exposure groups; though they did find significantly higher median free T3 concentrations among smoking mothers as well as significantly lower cord serum TSH concentrations in babies born to smoking mothers compared to those whose mothers were nonsmokers.

Active smoking is also associated with changes in PRL concentrations, but studies have had differing results. One study found a significant increase in PRL concentrations among men who were active smokers compared to nonsmokers (Xue et al., 2010). Two other studies reported increases and decreases, respectively, in PRL concentrations among animals exposed to tobacco smoke (Ng et al., 2006; Muraki et al., 1979).

Data is limited on the effects of secondhand tobacco smoke (STS) exposure on circulating TSH and PRL. Several studies have shown that exposure can disrupt the thyroid (Carrillo et al., 2009; Soldin et al., 2009; Flouris et al., 2008), but to our knowledge no studies to date have examined the relationship between STS exposure and PRL concentrations. Thus, the present study is intended to increase our understanding of the relationship between STS exposure and circulating TSH and PRL. We hypothesized that STS exposure is associated with increased serum levels of PRL and decreased serum TSH.

\section{Methods}

\subsection{Study population}

Subjects for the present study are a subset of a larger study examining predictors of in vitro fertilization (IVF) success, including STS exposure, and have been previously described (Meeker et al., 2007; Cramer et al., 2003). Briefly, in the larger study, couples undergoing IVF or intracytoplasmic sperm injection (ICSI) between 1994-1998 (study 1) and 1999-2003 (study 2) were recruited through three Boston-area clinics. Protocols were approved by the Human Research Committees at Brigham and Women's Hospital, the Harvard School of Public Health, and the University of Michigan. Approximately 65\% of couples approached agreed to participate in the study. Couples excluded from the study were those who were gestational carriers or who underwent gamete intra-fallopian transfer (GIFT), as well as those who required donor oocytes or donor semen. After exclusions, 2,350 couples who underwent from one to six IVF/ICSI treatment cycles were enrolled in the parent study. A self-administered questionnaire was used to obtain information from each subject on medical history and lifestyle factors such as: demographics, ages of both male and female partner, medical and reproductive history, smoking history, and duration of infertility. Information on IVF treatment and outcome was abstracted from clinical records. 314 nonsmoking patients for whom a blood sample was analyzed for PRL and TSH and for whom first-treatment-cycle follicular fluid (FF) was analyzed for cotinine were included in the present analysis.

\subsection{Hormone measurement}

When possible, a basal blood sample was collected from study participants. This sample was taken sometime during days one through five of the menstrual cycle and designated as the 
"true baseline." When a blood sample timed with the menses could not be collected, a sample was collected before IVF treatment began and was designated the "initial" specimen. Samples were aliquoted and stored at -80 degrees Centigrade. PRL and TSH were measured in archived serum samples using the AxSYM Immunoassay system (Abbott Diagnostics, Chicago, IL), which was described previously (Cramer et al., 2003). Briefly, the tests for PRL and TSH are solid-phase double antibody enzyme immunoassays employing microparticle enzyme immunoassay (MEIA) technology. For PRL, the limit of detection (LOD) was $0.6 \mathrm{ng} / \mathrm{ml}$ and assay performance was monitored using three quality control sera (Abbott Diagnostics). The coefficients of variation (CV) for PRL in the three control sera were $8.3,6.8$, and $4.8 \%$. TSH was analyzed using the MEIA technology (Ultrasensitive hTSH II). TSH levels were quantified as $\mu \mathrm{IU} / \mathrm{ml}$ based on assay calibrators standardized using the World Health Organization TSH $80 / 558$. The LOD was $0.03 \mu \mathrm{IU} / \mathrm{ml}$. TSH assay performance was also monitored using three quality control sera and the $\mathrm{CV}$ were 7.1, 6.2, and $7.4 \%$.

\subsection{Cotinine measurement}

Physicians and technicians were asked to retain FF during egg retrieval for each IVF cycle. FF was aspirated from follicles using a 16-gauge needle and constant suction from a Rocket pump apparatus. Fluid was collected from the largest visible follicle before using any flushing medium and then transferred to a sterile Petri dish. Oocytes were scanned for and removed. The fluid, normally discarded at this point, was placed into a $15 \mathrm{ml}$ conical tube and centrifuged for 15 minutes. The supernatant was placed into a clean storage tube, labeled, refrigerated, and transferred to the Brigham and Women's Hospital laboratory within 12 hours. At the laboratory, the specimens were aliquoted into 1 to $61.5 \mathrm{ml}$ tubes, depending upon the volume collected, and frozen at -80 degrees centigrade. FF was analyzed for cotinine using an enzyme-linked immunosorbent assay (ELISA; BioQuant, Inc., San Diego, CA). This single-step, competitive test uses spectrometric measurement to quantify cotinine in body fluids. It has a lower reporting limit of $0.3 \mathrm{ng} / \mathrm{ml}$ and inter- and intra-assay variations of four and six percent, respectively.

\subsection{Statistical Methods}

Data analysis was performed using SAS software (version 9.2; SAS Institute Inc., Cary, NC). Quantified cotinine concentrations below the LOD were kept as the reported value. Unquantified cotinine concentrations were assigned a value of one half of the LOD. All TSH and PRL levels were quantified and those below the LOD were also kept as the reported value.

Methods for categorizing tobacco smoke exposure have been previously described (Benedict et al., 2011a). Briefly, participants with FF cotinine concentrations greater than or equal to $10 \mathrm{ng} / \mathrm{ml}$ were classified as active smokers and excluded from further analysis, following Fuentes et al. (2010). Passive smokers, or STS-exposed nonsmokers, were those with FF cotinine concentrations less than $10 \mathrm{ng} / \mathrm{ml}$ and greater than $1.11 \mathrm{ng} / \mathrm{ml}$. This value was extended to our data from a urinary cotinine cutpoint (i.e. threshold) established by Zielińska-Danch et al. (2007). We defined unexposed nonsmokers as those with cotinine concentrations less than or equal to $1.11 \mathrm{ng} / \mathrm{ml}$. For comparison and to test sensitivity we also defined exposure based on the LOD of the cotinine assay. In this approach to classifying exposure, participants whose cotinine concentration was less than or equal to $0.15 \mathrm{ng} / \mathrm{ml}$ (1/2 the LOD) were considered unexposed. Those whose cotinine concentrations were greater than $0.15 \mathrm{ng} / \mathrm{ml}$ and less than $10 \mathrm{ng} / \mathrm{ml}$ were classified as exposed nonsmokers. Like the former approach, those with FF cotinine concentrations greater than or equal to 10 $\mathrm{ng} / \mathrm{ml}$ were considered active smokers and excluded. 
For all study participants, their serum sample was collected nearer in time to their firsttreatment-cycle FF sample compared to FF samples from later cycles. Thus, for the present study a subject's hormone concentrations were matched with the cotinine concentration from the FF sample collected during their first IVF treatment cycle. In preliminary analyses, Spearman correlation coefficients were calculated between cotinine, TSH, and PRL concentrations. Analysis of variance (ANOVA) was performed to determine if hormone levels differed between STS groups. PRL and TSH concentrations were skewed right and transformed by the natural logarithm for parametric analyses.

The relationships between exposure and outcome variables and key covariates were also examined to identify potential confounders. Multiple linear regression was then used to examine the relationship between hormone concentrations and STS exposure categories (i.e. STS-exposed vs. unexposed). Regression was first performed when using published cotinine cutpoints to classify exposure, followed by using the cotinine assay LOD, for comparison. Covariates considered for inclusion in adjusted models were age, body mass index (BMI), primary infertility diagnosis, date that the treatment cycle commenced, ovarian stimulation method, gonadotropin dose, study number, timing of blood sample collection, and ethnicity. Covariates were included in the final models depending on biological and statistical considerations (Hosmer \& Lemeshow, 1989). For example, PRL levels changed significantly with fertility diagnoses among study participants (data not shown). Also, moderately elevated TSH concentrations are frequently found in obese humans (Reinehr, 2010), hyperprolactinemia is associated with weight gain and obesity (Shibli-Rahhal \& Schlechte, 2009), and STS exposure may be associated with BMI (Braun et al., 2010; Kwok et al., 2010). Thus, BMI was included in adjusted models. We also included participant age and year of treatment in our final models since the study spanned 10 years (US population STS exposure levels have declined over time) and since PRL levels in women decrease steadily with age (Vekemans \& Robyn, 1975).

Due to the large number of non-detect cotinine concentrations in FF, we also examined the relationship between hormone levels and self-reported exposure. Self-reported exposure was obtained through the questionnaire. Participants were asked about their smoking behavior and STS exposure at home and at work and were considered exposed if they reported any STS exposure. The same covariates were included in both sets of regression models (i.e. when categorizing exposure based on FF cotinine and self-report) to maintain consistency.

\section{Results}

Among 314 nonsmoking participants, 27 percent had first cycle cotinine concentrations above the LOD. Hormone concentrations were quantified in all women. In preliminary analyses, we found a significant difference $(p=0.05)$ in PRL concentrations between STSexposed nonsmokers and unexposed nonsmokers based on FF cotinine concentrations (Table 1). There was a suggestive correlation between cotinine in FF and serum PRL (data not shown) but this analysis was limited by the high proportion of non-detectable levels of cotinine. This correlation was significant when restricting the analysis to women with detectable cotinine concentrations in FF.

In regression models adjusted for covariates, we observed a significant increase in PRL levels $(p=0.03)$ among STS-exposed nonsmokers compared to unexposed nonsmokers based on published FF cotinine cutpoints (Table 2). However, only a suggestive difference in PRL $(p=0.10)$ was observed between exposure groups when using the cotinine assay LOD to categorize exposure. No difference in TSH levels was observed between groups ( $p$ > $0.4)$. 
Because of the large proportion of non-detect FF cotinine concentrations and because cotinine concentrations measured at a given time may only reflect recent exposure to tobacco smoke, we tested the observed increase in PRL levels among STS-exposed nonsmokers through self-reported STS exposure. Using self-report to categorize exposure, we found a suggestive increase $(p=0.09)$ in PRL among those who reported exposure to STS compared to women who did not report exposure (Table 3). Similar to the analysis using FF cotinine concentrations, no significant change in TSH was observed when using self-report to categorize STS exposure.

\section{Discussion}

The aim of the present study was to explore the relationship between STS exposure and serum PRL and TSH concentrations in nonsmoking women who participated in a study on predictors of IVF success. As far as we are aware, this is the first study examining the effects of STS exposure on circulating PRL levels in humans. In adjusted models, we observed a significant increase in PRL concentrations among women exposed to STS compared to those unexposed based on cotinine concentrations measured in FF. The relationship between STS exposure and PRL was stronger when using a published cutpoint compared to using the cotinine analytical method LOD to categorize exposure. To further assess the results of these analyses, we conducted a sub-analysis using self-report to categorized exposure. There was a statistically suggestive increase in circulating PRL levels among subjects who self-reported STS exposure, but the association was weaker compared to the use of cotinine concentrations in FF to categorize exposure. The stronger relationships observed when using published cotinine cutpoints, compared to the cotinine method LOD or self-reported exposure, suggests it was the exposure categorization approach associated with the least amount of misclassification.

Similar to the findings of the present study, Xue et al. (2010) recently reported that tobacco smoke exposure, measured through plasma nicotine, was correlated with increased PRL levels $(\mathrm{r}=0.53 ; p<0.05)$. However, that study was conducted among men who were active smokers. Conversely, in another human study, Mello et al. (2001) reported that smoking mothers have reduced breast milk production and shorter lactation periods, findings which suggest that exposure to tobacco smoke may reduce PRL levels.

Animal studies on tobacco smoke and PRL have had conflicting results, as well. Decreased PRL levels were observed in female rats exposed to tobacco smoke from one to four cigarettes over a 90-minute period (Andersson et al., 1988). A study of the effects of tobacco smoke on gestational hormone levels among pregnant mice, however, found a suggestive increase $(p=0.07)$ in PRL levels among mice exposed to mainstream cigarette smoke compared to those that were unexposed ( $\mathrm{Ng}$ et al., 2006).

Tobacco smoke-induced changes in PRL levels may depend on species and/or gender (Shaw $\&$ al'Absi, 2010; Andersson et al., 1988), which could explain some of the conflicting results in the literature. In addition, endocrine responses to tobacco smoke exposure may differ depending on the duration, frequency and magnitude of exposure. Fuxe et al. (1989) reported that the initial effects of nicotine are characterized by a marked hypersecretion of PRL (which rapidly disappears) and that PRL secretion is inhibited with acute, intermittent nicotine treatment or exposure to cigarette smoke. This reduced PRL secretion due to tobacco smoke exposure is found mainly in chronic, habitual smokers.

The rapid disappearance of the acute stimulatory effects of nicotine may be due to a desensitization of central nicotinic cholinergic receptors from higher-level exposure during active smoking. Evidence also indicates that the inhibitory effects of nicotine on PRL 
secretion are produced through an activation of dopamine neurons by nicotinic receptors (Fuxe et al., 1989). In other words, chronic and/or high-level nicotine exposure may induce the release of dopamine, which inhibits PRL secretion. On the other hand, STS exposure may not be sufficient to desensitize nicotinic cholinergic receptors and/or activate dopamine neurons, which could result in elevated PRL concentrations. It should also be noted that one or several of the many constituents of tobacco smoke other than nicotine may be responsible for the observed relationship between exposure and changes in PRL secretion.

The findings of increased PRL concentrations among those exposed to STS versus those unexposed may have implications for a range of conditions. For example, hyperprolactinemia may be a cause of infertility in women with endometriosis (Gregoriou et al., 1999). In addition, epidemiology studies recently reviewed by Bernichtein et al. (2010) indicate that high levels of circulating PRL may be a risk factor for breast cancer. Data from two large prospective case-control studies (Nurses' Health Studies; NHS and NHS II) demonstrated a $40 \%$ increase in breast cancer risk for pre-menopausal women with PRL concentrations in the highest versus the lowest quartile of normal range ( $p$ trend $=0.05$; Bernichtein et al., 2010). A 30\% increase in breast cancer risk was observed among postmenopausal women $(p$ trend $=0.01$ ). There is also increasing evidence that locally-produced PRL (i.e. PRL expressed by human tissues other than the anterior pituitary, such as the mammary glands, the prostate, the skin, the brain and adipocytes) is associated with breast and prostate tumor growth (Bernichtein et al., 2010; Ben-Jonathan et al., 1996).

Studies have linked STS exposure with breast cancer, but there may not be sufficient evidence to infer a causal relationship. At least 21 studies have investigated the association between STS exposure and breast cancer risk among lifetime nonsmokers and these were recently reviewed (Centers for Disease Control and Prevention, 2006). Results of these studies varied, but considered collectively in a meta-analysis, breast cancer risk in lifetime nonsmokers was significantly associated with STS exposure. After stratification by menopausal status the association only remained significant among premenopausal women. Thus, it is possible that an association between PRL hypersecretion and STS exposure may increase breast cancer risk. If so, our results showing increased PRL in relation to STS exposure may provide important information regarding biological mechanisms in the discussion of previous reports of STS exposure and increased risk of breast cancer.

In the present study we did not observe an association between exposure to STS and serum TSH levels. No difference in TSH levels was found between exposure groups based on FF cotinine concentrations or based on self-reported exposure. Another study among 237 women aged 18-44 years, however, found a significant decrease $(p<0.05)$ in TSH concentrations among passive smokers compared to nonsmokers defined by serum cotinine (Soldin et al., 2009). The same study also reported a significant decrease $(p<0.01)$ in TSH levels in active smokers compared to nonsmokers, but no significant difference in TSH levels between active and passive smokers.

Iodine levels may influence the association between tobacco smoke exposure and thyroid hormone levels (Utiger 1995; Bertelsen \& Hegedüs, 1994). However, these effects have been examined only in active smokers compared to non-smokers and also most frequently in subjects with clinical thyroid disorders. The possibility of modulation of subclinical associations in passive smokers compared to unexposed non-smokers remains unexplored. Data on iodine levels were not available for our analysis. Future studies examining the relationship between STS and thyroid hormones may benefit from incorporating measures of this potential mediating variable. 
Because participants for the present study were IVF patients, the generalizability of our findings may be limited. For example, elevated levels of circulating PRL may be a cause of infertility (Wang et al., 2009). Thus, IVF patients may tend to have higher levels of circulating PRL than the general population, regardless of STS exposure. Also, demographic characteristics of an IVF cohort are likely different from the general population. If PRL is associated with socioeconomic status, for example, this could limit our generalizability. Another potential limitation of the present study is a relatively small sample size. Future studies with a larger number of participants exposed to STS may be needed to support our findings.

A precise biomarker was used in the present study to estimate STS exposure. However, our choice of biomarker may have influenced our results. FF cotinine data was accessible from a larger study among these women on implantation failure and IVF success (Benedict et al., 2011b). Cotinine concentrations in FF represent the fraction of plasma cotinine that diffused through the blood-follicle barrier and largely depend on concentrations gradients between the blood plasma, interstitial fluid and cells surrounding the oocytes (Benedict et al., 2011a). Thus, future research could explore the relationship between PRL secretion and STS exposure based on markers of exposure (e.g. serum cotinine) that may be more biologically relevant. However, based on two previous reports, cotinine in serum and FF is highly correlated $\left(\mathrm{R}^{2}=0.95\right.$ and $\mathrm{R}^{2}=0.89$; Fuentes et al., 2010; Paszkowski, 1998). Thus, a high degree of tobacco smoke exposure measurement error in the present study was not likely. In addition, the strength of the relationship between PRL and STS exposure based on published cutpoints $(p=0.03)$ was stronger than the relationships observed when using the LOD or self-report to classify exposure ( $p=0.10$ and 0.09 , respectively), which suggests that using the cutpoints may have resulted in less exposure misclassification. Moreover, the suggestive increase we observed in PRL concentrations among self-reported STS exposed women compared to those reporting no STS exposure further supports our conclusions.

\section{Conclusions}

In conclusion, we did not observe a significant difference in TSH concentration among those exposed to STS compared to those who were unexposed. However, our results indicate that STS exposure is associated with increased circulating PRL. This finding could have large public health significance due to the range of downstream adverse health effects potentially related to altered PRL levels. Future studies are needed to confirm our results, identify biological mechanisms involved, and better define the potential clinical and public health implications.

\section{Acknowledgments}

This work was supported by the Flight Attendant Medical Research Institute (FAMRI) and the NIH National Institute for Environmental Health Sciences (R01 ES013967) and National Institute of Child Health and Development (R01 HD32153).

\section{Abbreviations}

$\begin{array}{ll}\text { (PRL) } & \text { Prolactin } \\ \text { (TSH) } & \text { Thyroid stimulating hormone } \\ \text { (T3) } & \text { Triiodothyronine } \\ \text { (T4) } & \text { Thyroxine } \\ \text { (STS) } & \text { Secondhand tobacco smoke }\end{array}$


(IVF) In vitro fertilization

(ICSI) Intracytoplasmic sperm injection

(GIFT) Gamete intra-fallopian transfer

(FF) Follicular fluid

(MEIA) Microparticle enzyme immunoassay

(LOD) Limit of detection

(CV) Coefficients of variation

(ELISA) Enzyme-linked immunosorbent assay

(ANOVA) Analysis of variance

(BMI) Body mass index

(NHS) Nurses' Health Studies

\section{References}

Andersson K, Eneroth P, Fuxe K, Hrfstrand A. Effects of acute intermittent exposure to cigarette smoke on hypothalamic and preoptic catecholamine nerve terminal systems and on neuroendocrine function in the diestrous rat. Naunyn Schmiedebergs Arch. Pharmacol. 1988; 337(2):131-139. [PubMed: 2966898]

Benedict M, Missmer S, Vitonis A, Cramer D, Meeker J. Cotinine concentrations in follicular fluid as a measure of secondhand tobacco smoke exposure in women undergoing in vitro fertilization: intermatrix comparisons with urine and temporal variability. Chemosphere. 2011a; 84(1):110-116. [PubMed: 21397293]

Benedict M, Missmer S, Vahratian A, Berry K, Vitonis A, Cramer D, Meeker J. Secondhand tobacco smoke exposure is associated with increased risk of failed implantation and reduced IVF success. Human Rerpod. 2011b; 26(9):2525-2531.

Ben-Jonathan N, Mershon JL, Allen DL, Steinmetz RW. Extrapituitary prolactin: Distribution, regulation, functions, and clinical aspects. Endocr. Rev. 1996; 17(6):639-669. [PubMed: 8969972]

Bernichtein S, Touraine P, Goffin V. New concepts in prolactin biology. J. Endocrinol. 2010; 206(1): 1-11. [PubMed: 20371569]

Bertelsen JB, Hegedüs L. Cigarette smoking and the thyroid. Thyroid. 1994; 4(3):327-331. [PubMed: 7833671]

Bole-Feysot C, Goffin V, Edery M, Binart N, Kelly PA. Prolactin (PRL) and its receptor: Actions, signal transduction pathways and phenotypes observed in PRL receptor knockout mice. Endocr. Rev. 1998; 19(3):225-268. [PubMed: 9626554]

Braun J, Daniels J, Poole C, Olshan A, Hornung R, Bernert J, et al. Prenatal environmental tobacco smoke exposure and early childhood body mass index. Paediatr. Perinat. Epidemiol. 2010; 24(6): 524-534. [PubMed: 20955230]

Carrillo A, Metsios G, Flouris A. Effects of secondhand smoke on thyroid function. Inflamm. Allergy Drug Targets. 2009; 8(5):359-363. [PubMed: 20025583]

Centers for Disease Control and Prevention. The health consequences of involuntary exposure to tobacco smoke: a report of the surgeon general. Centers for Disease Control and Prevention, Office on Smoking and Health; Atlanta, GA: 2006. Retrieved from: http:// www.surgeongeneral.gov/library/secondhandsmoke/

Cramer DW, Sluss PM, Powers RD, McShane P, Ginsburgs ES, Hornstein MD, et al. Serum prolactin and TSH in an in vitro fertilization population: Is there a link between fertilization and thyroid function? J. Assist. Reprod. Genet. 2003; 20(6):210-215. [PubMed: 12877251] 
Flouris A, Metsios G, Jamurtas A, Koutedakis Y. Sexual dimorphism in the acute effects of secondhand smoke on thyroid hormone secretion, inflammatory markers and vascular function. Am. J. Physiol. Endocrinol. Metab. 2008; 294(2):E456-E462. [PubMed: 18073318]

Fuentes A, Muoz A, Barnhart K, Argello B, Daz M, Pommer R. Recent cigarette smoking and assisted reproductive technologies outcome. Fertil. Steril. 2010; 93(1):89-95. [PubMed: 18973890]

Fuxe K, Andersson K, Eneroth P, Hrfstrand A, Agnati LF. Neuroendocrine actions of nicotine and of exposure to cigarette smoke: Medical implications. Psychoneuroendocrinology. 1989; 14(1-2):1941. [PubMed: 2660182]

Gregoriou G, Bakas P, Vitoratos N, Papadias K, Goumas K, Chryssicopoulos A, et al. Evaluation of serum prolactin levels in patients with endometriosis and infertility. Gynecol. Obstet. Invest. 1999; 48(1):48-51. [PubMed: 10394092]

Hosmer, D., Jr; Lemeshow, S. Applied Logistic Regression. John Wiley \& Sons; New York: 1989. Model building strategies and methods for logistic regression; p. 82-134.

Krassas GE, Poppe K, Glinoer D. Thyroid function and human reproductive health. Endocr. Rev. 2010; 31(5):702-755. [PubMed: 20573783]

Kwok M, Schooling CM, Lam T, Leung G. Paternal smoking and childhood overweight: Evidence from the Hong Kong "children of 1997. Pediatrics. 2010; 126(1):e46-e56. [PubMed: 20587672]

McDonald S, Walker M, Ohlsson A, Murphy K, Beyene J, Perkins S. The effect of tobacco exposure on maternal and fetal thyroid function. Eur. J. Obstet. Gynecol. Reprod. Biol. 2008; 140(1):38-42. [PubMed: 18395957]

Meeker JD, Missmer SA, Cramer DW, Hauser R. Maternal exposure to second-hand tobacco smoke and pregnancy outcome among couples undergoing assisted reproduction. Human Reprod. 2007; 22(2):337-345.

Mello PR, Pinto GR, Botelho C. The influence of smoking on fertility, pregnancy and lactation. J. Pediatr. (Rio. J.). 2001; 77(4):257-264. [PubMed: 14647856]

Muraki T, Tokunaga Y, Nakadate T, Kato R. Inhibition by cholinergic agonists of the prolactin release induced by morphine. Naunyn Schmiedebergs Arch. Pharmacol. 1979; 308(3):249-254. [PubMed: 503252]

$\mathrm{Ng} \mathrm{S}$, Steinetz B, Lasano S, Zelikoff J. Hormonal changes accompanying cigarette smoke-induced preterm births in a mouse model. Exp. Biol. Med. 2006; 231(8):1403-1409.

Paszkowski T. Concentration gradient of cotinine between blood serum and preovulatory follicular fluid. Ginekol. Pol. 1998; 69(12):1131-1136. [PubMed: 10224789]

Reinehr T. Obesity and thyroid function. Mol. Cell Endocrinol. 2010; 316(2):165-171. [PubMed: 19540303]

Shaw D, al'Absi M. Blunted opiate modulation of prolactin response in smoking men and women. Pharmacol. Biochem. Behav. 2010; 95(1):1-5. [PubMed: 19969014]

Shibli-Rahhal A, Schlechte J. The effects of hyperprolactinemia on bone and fat. Pituitary. 2009; 12(2):96-104. [PubMed: 18338266]

Shields B, Hill A, Bilous M, Knight B, Hattersley A, Bilous R, et al. Cigarette smoking during pregnancy is associated with alterations in maternal and fetal thyroid function. J. Clin. Endocrinol. Metab. 2009; 94(2):570-574. [PubMed: 19017761]

Soldin O, Goughenour B, Gilbert S, Landy H, Soldin S. Thyroid hormone levels associated with active and passive cigarette smoking. Thyroid. 2009; 19(8):817-823. [PubMed: 19505184]

Utiger RD. Cigarette smoking and the thyroid. N. Engl. J. Med. 1995; 333(15):1001-1002. [PubMed: 7666896]

Vekemans M, Robyn C. Influence of age on serum prolactin levels in women and men. BMJ. 1975; 4(5999):738-739. [PubMed: 1212585]

Wang H, Gorpudolo N, Behr B. The role of prolactin- and endometriosis-associated infertility. Obstet. Gynecol. Surv. 2009; 64(8):542-547. [PubMed: 19624865]

Xue Y, Morris M, Ni L, Guthrie S, Zubieta J, Gonzalez K, et al. Venous plasma nicotine correlates of hormonal effects of tobacco smoking. Pharmacol. Biochem. Behav. 2010; 95(2):209-215.

[PubMed: 20100507] 
Zieliń;ska-Danch W, Wardas W, Sobczak A, Szoltysek-Boldys I. Estimation of urinary cotinine cutoff points distinguishing non-smokers, passive and active smokers. Biomarkers. 2007; 12(5):484496. [PubMed: 17701747] 


\section{Research highlights}

- Women studied were non-smokers who sought in vitro fertilization treatment

- Prolactin was positively associated with secondhand tobacco smoke

- Thyroid stimulating hormone was not associated with secondhand tobacco smoke

- Future studies for confirmation of results are needed 


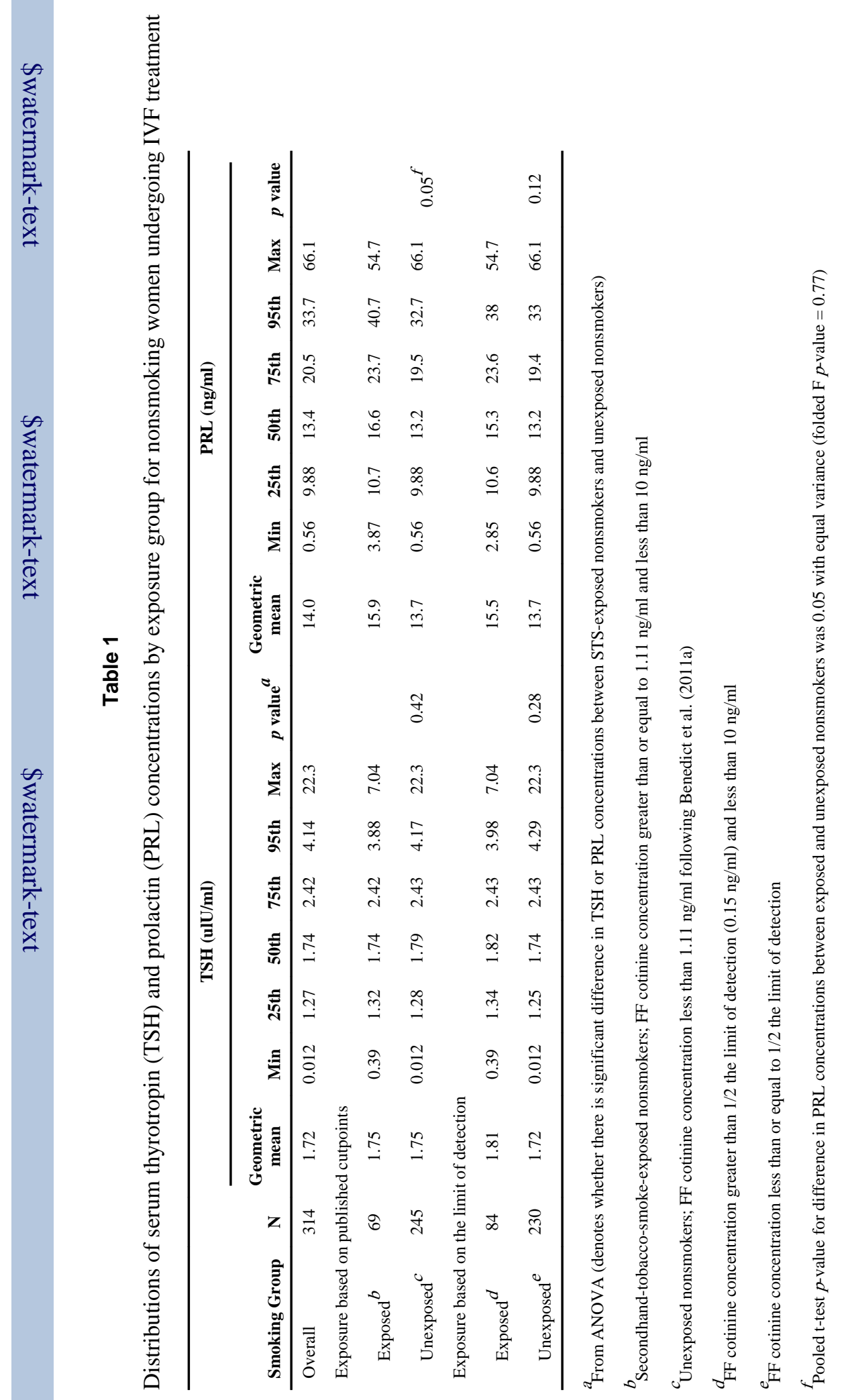

Environ Toxicol Pharmacol. Author manuscript; available in PMC 2013 November 01. 







\section{Table 3}

Adjusted ${ }^{a}$ regression coefficients and $95 \%$ confidence intervals for change in ln-serum hormone concentrations associated with self-reported secondhand tobacco smoke exposure ${ }^{b}$ among 314 nonsmoking women undergoing IVF treatment

\begin{tabular}{lccc}
\hline & $\begin{array}{c}\text { Parameter } \\
\text { estimate }\end{array}$ & 95\% CI & p-value \\
\cline { 2 - 4 } TSH & -0.14 & $-0.35,0.072$ & 0.20 \\
Prolactin & 0.16 & $-0.026,0.35$ & 0.09 \\
\hline
\end{tabular}

${ }^{a}$ Adjusted for age, BMI, primary fertility diagnosis and year of IVF treatment

$b_{\text {Number reporting secondhand tobacco smoke exposure }=40}$ 